\title{
Método dos Mínimos Quadrados com Regressão Wavelets para Redução de Erros no Processamento de Sinais GPS ${ }^{1}$
}

E.M. de SOUZA 2 , J.F.G. MONICO ${ }^{3}$, A. PAGAMISSE, W.G.C. POLEZEL ${ }^{5}$, FCT, UNESP, 19060-900 Presidente Prudente, SP, Brasil.

Resumo. O sistema de posicionamento por satélite GPS tem revolucionado a navegação, trazendo praticidade, precisão e economia para diversas aplicações, desde a navegação automobilística, aplicações na agricultura e até mesmo a aviação. Embora precisões muito boas possam ser atingidas, existem erros que prejudicam a qualidade do posicionamento, tais como o efeito do multicaminho, devido às reflexões do sinal. Embora a seleção cuidadosa do local da antena e o uso de antenas e receptores especiais possam minimizar o multicaminho, este efeito, em geral, não é eliminado e, frequentemente, permanece como um dos erros mais significativos nos resultados GPS. O multicaminho de freqüência alta devido aos atrasos longos (refletores/superfícies longe do receptor) pode ser atenuado por métodos de redução de ruído. Mas o multicaminho de freqüência baixa devido aos atrasos curtos (refletores/superfícies perto do receptor) é muito difícil de ser reduzido ou modelado. Neste artigo é proposto um método inovador baseado em regressão wavelets que pode detectar e reduzir efetivamente o efeito do multicaminho. Foi realizado um experimento com objetos refletores próximos ao receptor para causar, principalmente, multicaminho de baixa freqüência. Os resultados foram comparados com e sem a redução do multicaminho utilizando regressão wavelets. Após a atenuação do multicaminho, o posicionamento ficou até $73 \%$ mais acurado, ou seja, erros de $0,022 \mathrm{~m}$ foram reduzidos para $0,006 \mathrm{~m}$.

\section{Introdução}

Para realizar o processamento de sinais do Sistema de Posicionamento Global GPS, usualmente utiliza-se a estimativa recursiva pelo Método dos Mínimos Quadrados (MMQ). Para tanto, a formulação do modelo matemático, o qual consiste das partes funcional e estocástica, é essencial. Se o modelo funcional é adequado, os resíduos estimados devem ter comportamento randômico. Entretanto, os sinais GPS são contaminados por diversos tipos de erros, nem todos modeláveis. Dessa forma, obtêm-se resíduos que contêm, além da componente aleatória, as características

\footnotetext{
${ }^{1}$ Trabalho financiado pela FAPESP - Processos 03/12770-3 e 04/02645-0

${ }^{2}$ eniuce@yahoo.com.br, Programa de Pós Graduação em Ciências Cartográficas

${ }^{3}$ galera@fct.unesp.br, Departamento de Cartografia

${ }^{4}$ aylton@fct.unesp.br, Departamento de Matemática, Estatística e Computação

${ }^{5}$ wesleypolezel@hotmail.com, Departamento de Cartografia
} 
dos erros não modelados. É de interesse extrair ou minimizar os erros contidos nas medidas GPS. Com este intuito, no posicionamento relativo GPS em linhas de base curtas, ou seja, envolvendo pelo menos dois receptores que não distem mais de $15 \mathrm{~km}$ um do outro, a técnica de duplas diferenças (DD) é utilizada na construção do modelo funcional. Dessa forma, os erros relacionados com as órbitas dos satélites, com a influência da atmosfera e com a diferença entre os relógios do satélite e receptor são reduzidos. Porém, o mesmo não acontece com o efeito multicaminho, pois este efeito é causado pela reflexão do sinal GPS em superfícies nas proximidades de cada receptor. Devido ao maior percurso dos sinais refletidos, estes chegam atrasados em relação ao sinal direto. Geralmente, sinais refletidos em superfícies que não distem mais de $20 \mathrm{~m}$ do receptor são considerados com atrasos curtos, caracterizando efeitos do multicaminho de freqüência baixa e que causam os erros mais significativos. O multicaminho devido aos atrasos longos é de freqüência alta e causa erros menores. Isto faz com que o multicaminho dependa da geometria do ambiente de cada local em que se deseja determinar a posição e que não seja eliminado nas DD. Logo, o multicaminho se torna a fonte de erro mais significativa nos resíduos advindos do MMQ. Consequentemente, a qualidade do posicionamento é degradada devido a esse efeito. Isto tem requerido esforços de muitos pesquisadores em todo o mundo no sentido de redução deste efeito.

Neste sentido, diversas antenas e receptores foram desenvolvidos especialmente para reduzir o efeito do multicaminho. Entretanto, apenas a parte de freqüência alta desse efeito é geralmente atenuada [15]. Esse efeito pode também ser tratado no processamento dos dados. Devido às características espectrais do efeito do multicaminho, alguns métodos foram desenvolvidos para redução do mesmo utilizando a análise espectral. Uma técnica recente é baseada em um operador de redução de ruído no tempo e freqüência denominada Time Frequency Denoising Operator que envolve a Transformada de Fourier para redução do efeito do multicaminho [5]. Segundo os autores a técnica é promissora, mas a acurácia dos resultados não foi analisada. Um outro método, que funciona como um redutor de ruído, utiliza o MMQ reponderado (RLSCO - Reweighed Least Squares for Correlated Observations) [19]. Nesse método, um modelo de variância Fuzzy é utilizado para levar em consideração o multicaminho de freqüência alta, difração e ruídos. Outros métodos envolvem wavelets de uma forma ou de outra e têm provido bons resultados ([18], [10] e [11]).

Entretanto, ainda falta a concretização de uma técnica prática e eficiente para redução do efeito do multicaminho tanto de freqüência alta como de baixa. Foi mostrado por [13] que o efeito do multicaminho de freqüência alta devido aos atrasos longos pode ser atenuado por métodos de redução de ruído (denoising). Mas o efeito do multicaminho de freqüência baixa devido aos atrasos curtos é muito difícil de ser reduzido ou modelado. Neste artigo, é proposto um método para a redução efetiva do efeito do multicaminho, o qual envolve o uso da Regressão Wavelets (RW) no MMQ (MMQRW). Neste método, a RW é aplicada aos resíduos do MMQ para estimar as componentes dos erros do multicaminho tanto de alta como de baixa freqüência. Os efeitos de freqüência baixa, que são muito difíceis de serem detectados nas DD, podem ser estimados a partir dos resíduos das DD. Uma vez estimadas, as componentes dos erros do multicaminho são utilizadas para a correção 
deste efeito no modelo funcional, ou seja, nas observações das DD. Posteriormente, o MMQ recursivo é aplicado novamente utilizando as observações de DD corrigidas do efeito do multicaminho.

Assim, na seção 2 é apresentado o MMQRW para a redução do efeito do multicaminho. O experimento realizado, os resultados e análises são discutidos na seção 3. Finalmente, as considerações finais do trabalho são feitas na seção 4.

\section{MMQRW para Estimativa e Correção do Efeito do Multicaminho no Sinal GPS}

Para realizar o processamento dos sinais GPS, usualmente utiliza-se a estimativa por MMQ considerando o modelo linearizado $V=A X-L$, com $n>u$, onde $L$ é o vetor com $n$ observações de DD GPS, $X$ é o vetor das $u$ incógnitas ou parâmetros (coordenadas do posicionamento, dentre outras), $A$ é a matriz design $(n \times u)$ de escalares conhecidos e $V$ é o vetor dos $n$ resíduos das DD. O princípio do MMQ é que $\Phi=(A X-L)^{T} P(A X-L)$ seja mínimo, onde $P$ é a matriz dos pesos $(u \times u)$. Essa minimização proporciona a estimativa dos parâmetros $X=\left(A^{T} P A\right)^{-1}\left(A^{T} P L\right)$.

Devido ao fato de serem utilizadas as DD na construção do modelo funcional, apenas o efeito do multicaminho não é reduzido; pelo contrário, ele pode até aumentar, já que é um efeito altamente dependente da geometria do ambiente de cada receptor GPS. Logo, o multicaminho se torna a fonte de erro mais significativa nos resíduos $V$ das DD. Assim, a metodologia que está sendo proposta consiste em aplicar a Regressão Wavelets (RW) nestes resíduos para a estimativa do efeito do multicaminho. Uma vez estimado, este efeito é corrigido no sinal de DD GPS e o MMQ é aplicado novamente. Este procedimento é denotado por MMQRW.

Assim, considerando o modelo $V_{i}=f\left(t_{i}\right)-\varepsilon_{i}, i=1, \ldots, n$, onde $f$ é a função desconhecida do efeito do multicaminho e $\varepsilon_{i} \sim N(0,1)$ é uma variável aleatória gaussiana, representando o erro da medida, a RW consiste em:

1) Aplicar o algoritmo piramidal ([6]) aos resíduos $V_{i}$ das DD GPS para os coeficientes wavelet da Transformada Wavelets Discreta (TWD) como apresentado em [14]. Esse algoritmo permite que os componentes de alta freqüência (detalhes e ruídos) do sinal sejam separados dos componentes de baixa freqüência (multicaminho de baixa freqüência) em vários níveis de resolução. O nome multiresolução provém dessa idéia. Neste processo, uma wavelet mãe que constitui uma base ortonormal é escolhida. Foi utilizada a wavelet Symmlets com 8 momentos nulos, pois [12] mostrou que essa wavelet proporciona melhores resultados em aplicações GPS que outras Symmlets e wavelets de Daubechies. Os coeficientes dos filtros implementados em relação a essa wavelet podem ser encontrados em [2];

2) Modificar os coeficientes wavelet utilizando limiarização wavelet, em especial, o esquema de limiarização rígido e o limiar universal [3]. No primeiro nível de decomposição é calculado o nível de ruído do sinal de DD GPS para que os componentes relativos aos ruídos possam ser separados e os componentes relativos ao efeito do multicaminho possam ser detectados. Não é necessário modificar os componentes wavelet nos demais níveis de decomposição quando se trata de um sinal de DD proveniente do posicionamento relativo GPS envolvendo linhas de base 
curtas, pois, neste caso, o efeito do multicaminho é predominante nos resíduos das $\mathrm{DD}$, não necessitando ser separado de outros efeitos;

3) Reconstruir a função $f$ desejada (efeito do multicaminho) utilizando a TWDI dos coeficientes do estágio (2).

A parte crucial da RW é o passo 2 [9]. Praticamente todos os trabalhos relevantes em esquemas de limiarização e limiares ótimos estão contidos em [3] e [4]. Estes procedimentos também foram descritos em [13], logo, não serão apresentados neste artigo.

Após aplicar a RW e estimar o efeito do multicaminho, estes valores são corrigidos diretamente nas observações de DD, resultando em um vetor $L$ sem o efeito do multicaminho. Finalmente, o MMQ é aplicado novamente para que as coordenadas do posicionamento possam ser determinadas com melhor qualidade.

\section{Experimentos e Resultados}

Nesta seção são apresentados os resultados de um experimento realizado em Presidente Prudente durante os dias 16, 17, 20 e 21/09/03. Nos dias 16 e 17/09/03, um receptor Astech ZXII foi colocado a $6 \mathrm{~m}$ de uma carreta revestida de alumínio representando um objeto refletor de $2,5 \times 13 \mathrm{~m}$ e a $0,5 \mathrm{~m}$ de uma placa de zinco medindo $0,30 \times 1,10 \mathrm{~m}$ (Figura 1 ). Coletaram-se os dados em dois dias para verificação da repetibilidade do efeito do multicaminho, pois quando a geometria do levantamento permanece inalterada, este efeito muda de acordo com o movimento dos satélites e, consequentemente, deve apresentar o mesmo padrão durante dias consecutivos. Devido à distância curta dos objetos refletores em relação ao receptor, o principal efeito do multicaminho causado é de freqüência baixa, permitindo que o desempenho do método proposto seja avaliado em um caso de erros críticos provenientes do multicaminho. Nos dois últimos dias (20 e 21), os objetos foram removidos para que o cálculo das coordenadas "verdadeiras" pudesse ser estimado.

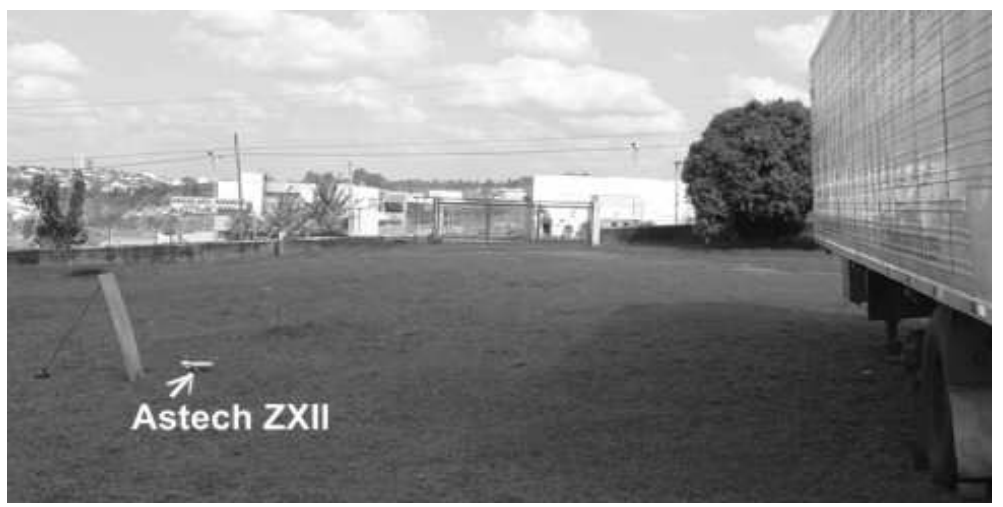

Figura 1: Cenário do experimento realizado nos dias 16 e 17/09/03

As DD das observáveis do GPS (chamadas de código e fase), coletadas no experimento, foram processadas no software GPSeq, em desenvolvimento na FCT/UNESP. 
As medidas de código e fase são obtidas, respectivamente, a partir da correlação do código e diferença de fase, gerados pelo satélite no instante de transmissão e suas réplicas geradas no receptor no instante de recepção.

Antes de aplicar a metodologia proposta, é importante verificar se os erros nos resíduos são realmente de multicaminho. Para tanto, os resíduos das DD obtidos a partir do MMQ foram analisados nos dias 16 e 17/09/03 (Figura 2).

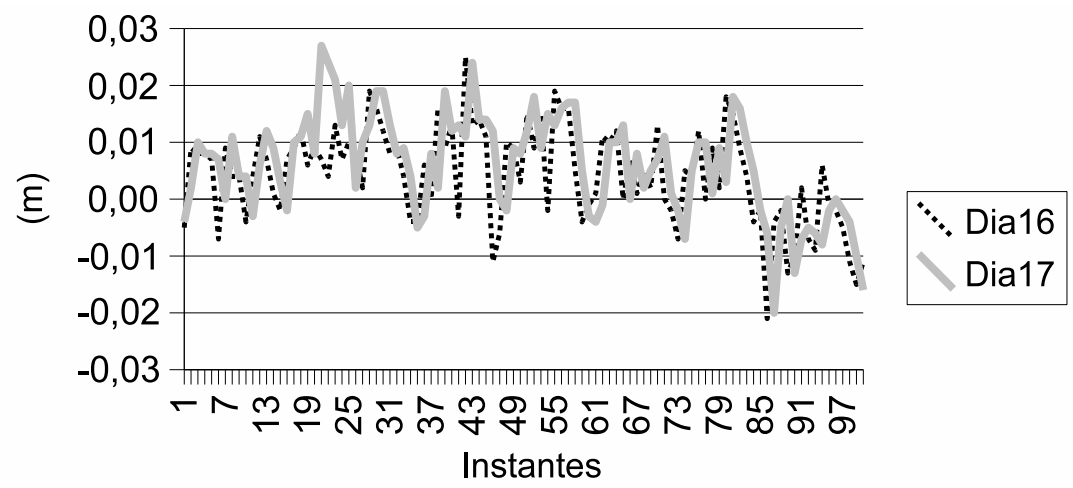

Figura 2: Repetibilidade do multicaminho nos resíduos das DD

Pode-se observar na figura 2 que os resíduos são parecidos nos dois dias, apresentando um comportamento similar. Logo, tem-se um exemplo da repetibilidade do multicaminho nos dois dias analisados, indicando a presença e predominância do efeito do multicaminho. O mesmo foi verificado para as demais DD. Uma vez verificada a repetibilidade, o MMQRW também foi aplicado para redução do efeito do multicaminho e os resultados foram comparados.

Os resíduos das DD do código e da fase para os casos com multicaminho menos (DD23-15) e mais (DD23-21) acentuado são ilustrados nas figuras 3 a 6 . A notação DD23-15, por exemplo, refere-se a DD entre os sinais dos satélites 23 e 15.

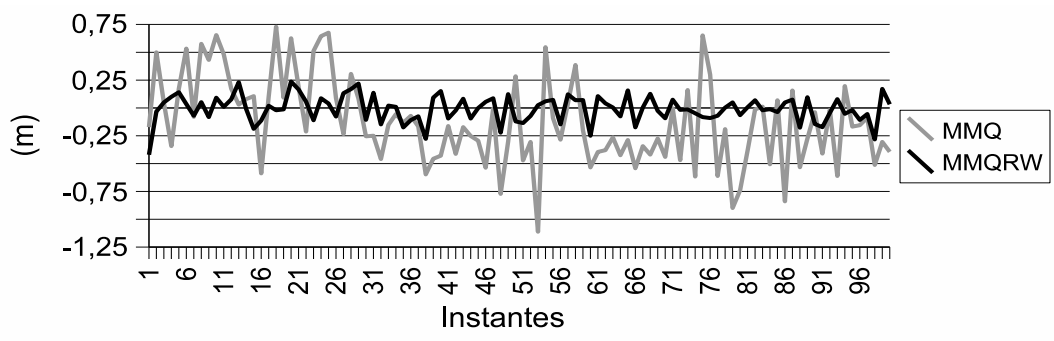

Figura 3: Resíduos da DD23-15 para o código - Multicaminho menos acentuado

A partir das figuras 3 a 6 pode-se verificar que a presença do efeito do multicaminho foi significativamente reduzida aplicando o MMQRW. Este fato foi confirmado para todas as DD, atingindo até $99 \%$ de melhoria no Erro Médio Quadrático 


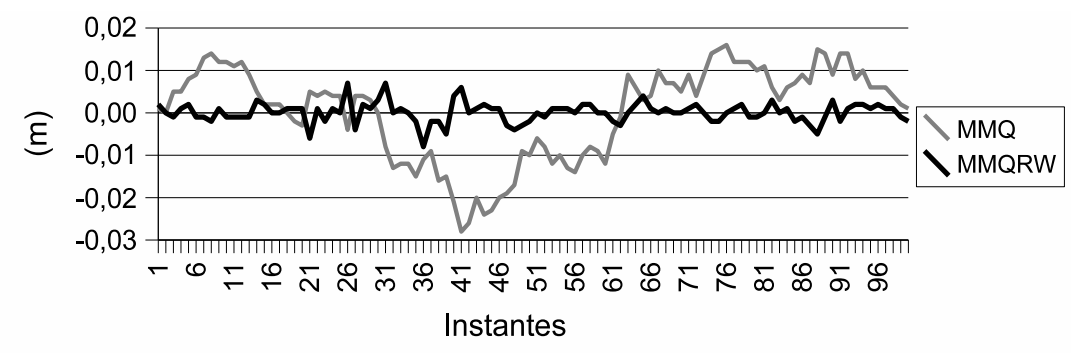

Figura 4: Resíduos da DD23-15 para a fase - Multicaminho menos acentuado

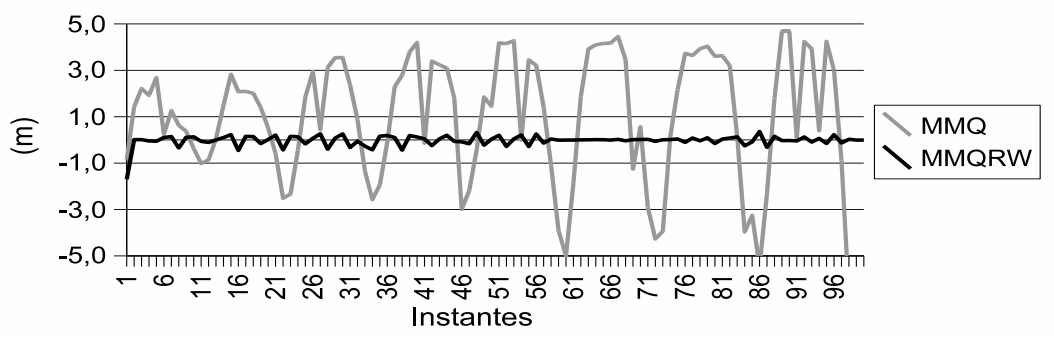

Figura 5: Resíduos da DD23-21 para o código - Multicaminho mais acentuado

(EMQ) para o código e até $90 \%$ na fase.

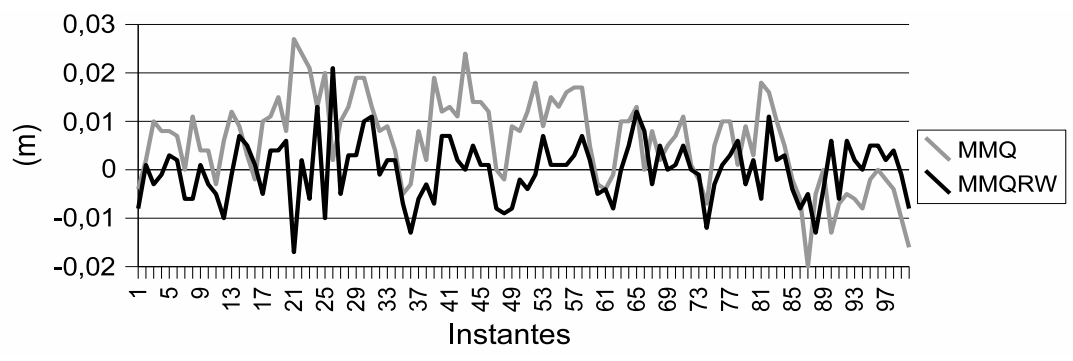

Figura 6: Resíduos da DD23-21 para a fase - Multicaminho mais acentuado

Para comparar a qualidade das observações de DD para a pseudodistância e fase, antes (MMQ) e após (MMQRW) a correção do efeito do multicaminho, utilizouse o teste estatístico GOM (Global Overall Model) [16]. Os valores obtidos com esta análise podem ser vistos na figura 7a. No posicionamento GPS, para que as coordenadas sejam determinadas, é necessário estimar o número inteiro de ciclos da observação de fase, denominado ambiguidade. Foi implementado também no software GPSeq um teste estatístico RIA (Ratio Integer Aperture), desenvolvido por [17], que possibilita estimar com confiança a probabilidade de sucesso da solução das ambiguidades (Figura 7b).

Verifica-se a partir da figura 7a que o valor do teste estatístico GOM nos dias 
(a)

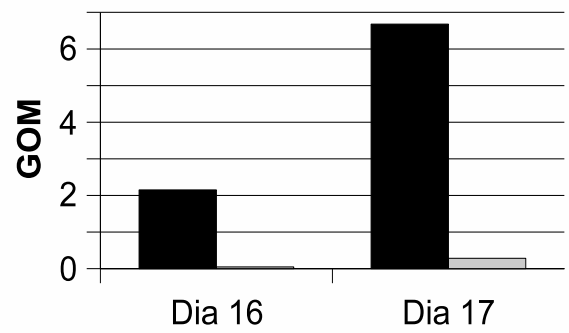

(b)

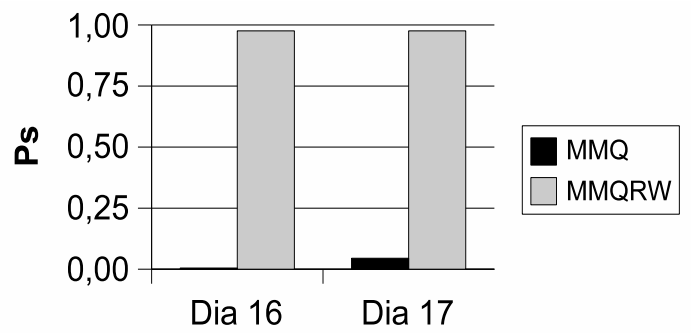

Figura 7: (a) Teste estatístico GOM (b) Teste Estatístico RIA

16 e 17 é bem menor para o MMRW, mostrando que o efeito do multicaminho foi minimizado, atingindo até $98 \%$ de melhoria. A partir da figura $7 \mathrm{~b}$, observa-se que antes da correção do efeito do multicaminho, a probabilidade de sucesso (Ps) estava muito baixa. Isso é esperado pelo fato de o efeito do multicaminho prejudicar muito a solução das ambigüidades. Após a correção deste efeito, o teste RIA indicou que as ambigüidades foram fixadas corretamente com quase $98 \%$ de probabilidade. Logo, tem-se novamente indicativos da eficiência do MMQRW detecção e correção do efeito do multicaminho a partir dos resíduos das DD GPS.

As coordenadas antes e após a correção do multicaminho foram comparadas com as coordenadas consideradas "verdadeiras". As discrepâncias entre as coordenadas são apresentadas na figura 8a e seus respectivos desvios padrão na figura 8b.
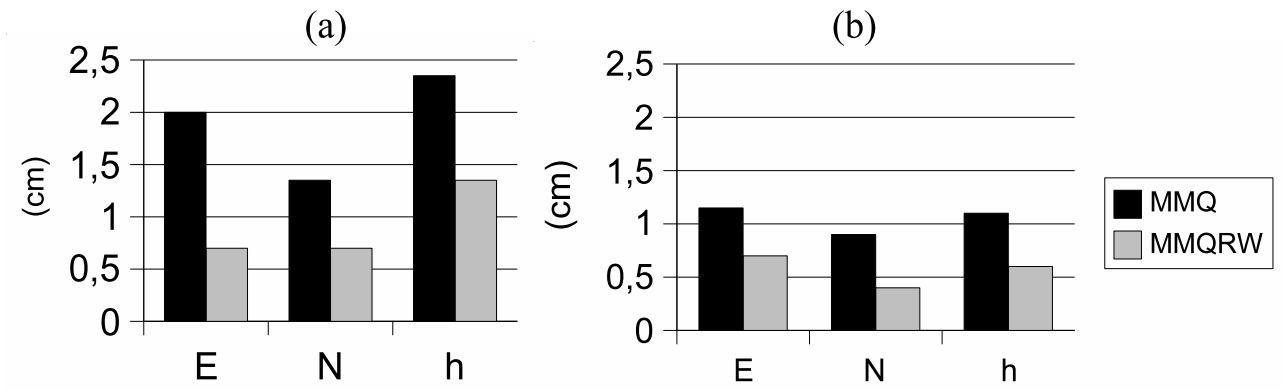

Figura 8: (a) Discrepâncias entre as coordenadas referentes as componentes E, $\mathrm{N}$ e h de uma sistema de referência ortogonal local (b) Desvio Padrão das Coordenadas

A partir da figura 8 nota-se que tanto as discrepâncias entre as coordenadas, como o desvio padrão das mesmas, diminuiram muito, indicando que após a redução do multicaminho utilizando o MMWQRW, as coordenadas estão mais acuradas. As discrepâncias calculadas na figura 8a envolvem resultados dos dias 16 e 17 . Na componente E das coordenadas no dia 16, obteve-se até $73 \%$ de melhoria, ou seja, erros de 0,022 $\mathrm{m}$ foram reduzidos para 0,006 $\mathrm{m}$. Na média de todas as componentes 
envolvendo os dois dias de dados processados, obteve-se $52 \%$ de melhoria.

\title{
4. Conclusão
}

Conclui-se a partir dos resultados apresentados que o MMQRW é muito eficiente na redução do efeito do multicaminho em aplicações GPS. A presença do efeito do multicaminho de baixa freqüência nos resíduos das DD foi significativamente reduzida. O EMQ dos resíduos melhorou até $99 \%$ utilizando o MMQRW. A acurácia das coordenadas atingiu até $73 \%$ de melhoria. Trata-se de resultados muito importantes, pois está reconhecido internacionalmente, conforme trabalhos citados neste artigo e vários outros, que se deve direcionar esforços no sentido de reduzir ou eliminar o efeito do multicaminho para que seja possível realizar aplicações de alta precisão, utilizando o GPS em qualquer ambiente.

\begin{abstract}
The GPS satellite positioning system has revolutionized the navigation, bringing facility, precision and economy for several applications, since automobile navigation, agriculture and even the aviation. Although very good precisions can be reached, there are errors that degrade the quality of the positioning, such as the multipath effect due to reflections of the signal. Although the careful selection of antenna site and the use of especial antennas and receivers can minimize multipath, it cannot always be eliminated and frequently remains as one of the more significant errors in the GPS results. It was shown that the high-frequency multipath from large delays (reflectors/surfaces far from the receiver) can be attenuated by denoising methods. But the low-frequency multipath from short delays (reflectors/surfaces near the receiver) is very difficult to be reduced or modeled. In this paper, an innovator method is proposed which is based on wavelet regression, which can effectively detect and reduce the low-frequency multipath. An experiment was carried out using objects placed close to the receiver in order to cause, mainly, lowfrequency multipath. The results with and without the multipath mitigation using wavelet regression were compared. After mitigating the multipath, the positioning became up to $73 \%$ more accurate where errors of $0.022 \mathrm{~m}$ were reduced to $0.006 \mathrm{~m}$.
\end{abstract}

\section{Referências}

[1] C.K. Chui, "An Introduction to Wavelets", Academic Press, Boston, 1992.

[2] I. Daubechies, "Ten Lectures on Wavelets". SIAM, Philadelphia, PA, 1992.

[3] D.L. Donoho, I.M. Johnstone, Ideal spatial adaptation by Wavelet Shrinkage. Biometrika, 81 (1994), 425-455.

[4] D.L. Donoho et al., Wavelet Shrinkage: asymptopia? (with discussion), Journal Royal Statistical Society, 57 (1995), 301-369.

[5] A. Jarrot, C. Ioana, A. Quinquis, J.C.L. Gac, Multi-component signal denoising using unitary time-frequency transforms, in "Proceedings of EUSIPCO", Antalya, Turkey, 2005. 
[6] S. Mallat, "A Wavelet Tour of Signal Processing", Academic Press, San Diego, CA, 1998 .

[7] J.F.G. Monico, "Posicionamento pelo NAVSTAR - GPS: Descrição, Fundamentos e Aplicações", UNESP, São Paulo, 2000.

[8] P.A. Morettin, "Ondas e Ondaletas: da Análise de Fourier à Análise de Ondaletas", EDUSP, São Paulo, 1999.

[9] G.P. Nason, "Wavelet Regression by Cross-Validation". TR 447, Dept. Statistics, Stanford University, 1994.

[10] C. Satirapod, C. Ogaja, J. Wang, C. Rizos. An approach to GPS analysis incorporating wavelet decomposition. Artificial Satellites, 36 (2001), 27-35.

[11] E.M. Souza, "Efeito de Multicaminho de Alta Frequência no Posicionamento Relativo GPS Estático: Detecção e Atenuação utilizando Wavelets". Dissertação de Mestrado, FCT/UNESP, Presidente Prudente, SP, 2004.

[12] E.M. Souza, J.F.G. Monico, Wavelet Shrinkage: High frequency multipath reduction from GPS relative positioning. GPS Solutions, 8 (2004), 152-159.

[13] E.M. Souza, A. Pagamisse, M. Meneguette Jr, J.F.G. Monico, Processamento de sinais com wavelets: redução do efeito do multicaminho do sinal GPS, em "Seleta do XXVII CNMAC" (E.X.L. de Andrade, J.M. Balthazar, S.M. Gomes, G.N. Siva e A. Sri Ranga, eds.), TEMA - Tendências em Matemática Aplicada e Computacional, Vol. 6, No. 1, pp. 177-186, SBMAC, 2005.

[14] E.M. Souza, A. Pagamisse, J.F.G. Monico, W.G.C. Polezel, Comparação das bases de wavelets ortonormais e biortogonais: implementação, vantagens e desvantagens no posicionamento com GPS, em "Seleta do XXIX CNMAC" (C.F. Bracciali, L.M. Carvalho, A. Castelo e A.J. Silva Neto, eds.), TEMA - Tendências em Matemática Aplicada e Computacional, Vol. 8, No. 1, pp. 149-158, SBMAC, 2007.

[15] D.B. Taille, J. Maenpa, H.J. Eules, P. Cross, Overcoming the limitations of the phase multipath mitigation window, in "Proceedings of 16th ION GPS", Portland, ORE. 2003.

[16] P.J.G. Teunissen, Quality control and GPS, in "GPS for Geodesy" (P.J.G. Teunissen, A. Kleusberg, eds.) 2 ed. Berlin: Springer Verlage, pp. 271-318, 1998.

[17] P.J.G. Teunissen, S. Verhagen, On the foundation of the popular ratio test for GNSS ambiguity resolution, in "Proceedings of 17th ION GPS", Long Beach, CA. pp. 2529-2540, 2004.

[18] L. Xia, Approach for multipath reduction using wavelet algorithm, in "Proceedings of 17th ION GPS", Salt Lake City, UT. pp. 2134-2143, 2001.

[19] A. Wieser, F.K. Brunner, Short static GPS sessions: robust estimation results. GPS Solutions, 5, No. 3 (2002), pp. 70-79. 
